\title{
Aaron Moore, Constructing East Asia : technology, ideology and Empire in Japan's Wartime Era, 1931-1945
}

Stanford, Stanford University Press, 2013

\section{Aleksandra Kobiljski}

\section{(2) OpenEdition}

\section{Journals}

\section{Édition électronique}

URL : https://journals.openedition.org/artefact/7285

DOI : 10.4000/artefact.7285

ISSN : 2606-9245

Éditeur :

Association Artefact. Techniques histoire et sciences humaines, Presses universitaires du Midi

Édition imprimée

Date de publication : 18 juin 2015

Pagination : 223-226

ISBN : 978-2-271-08155-1

ISSN : 2273-0753

\section{Référence électronique}

Aleksandra Kobiljski, « Aaron Moore, Constructing East Asia : technology, ideology and Empire in Japan's Wartime Era, 1937-1945 », Artefact [En ligne], HS 01 | 2015, mis en ligne le 28 avril 2021, consulté le 05 mai 2021. URL : http://journals.openedition.org/artefact/7285 ; DOI : https://doi.org/10.4000/artefact. 7285

Ce document a été généré automatiquement le 5 mai 2021.

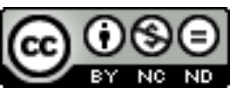

Artefact, Techniques, histoire et sciences humaines est mise à disposition selon les termes de la Licence Creative Commons Attribution - Pas d'Utilisation Commerciale - Pas de Modification 4.0 International. 


\section{Aaron Moore, Constructing East Asia: technology, ideology and Empire in Japan's Wartime Era, 1931-1945}

Stanford, Stanford University Press, 2013

\section{Aleksandra Kobiljski}

\section{RÉFÉRENCE}

Aaron Moore, Constructing East Asia : technology, ideology and Empire in Japan's Wartime Era, 1931-1945, Stanford, Stanford University Press, 2013, 328 p.

1 Constructing East Asia est une étude fine de ce que l'auteur appelle " l'imaginaire technologique » du Japon pendant les trente premières années de la période showa (1926-1989). La première partie du livre explore l'apparition du terme " technologie » (gijutsu 技術) dans le discours public et politique, tandis que la seconde explore l'émergence de concepts tels que « technologie globale » (sôgô gijutsu 総合技術) dans un échange dynamique entre les plans bien élaborés et les réalités complexes de l'ingénierie coloniale.

2 Le livre commence avec une analyse du " débat sur la théorie de la technologie » (gijitsuron ronsô 技術論論争) auquel ont surtout pris part des intellectuels marxistes qui tentaient de comprendre l'impact de la technologie sur la société japonaise moderne. Conscients des débats de l'époque en Europe et aux États-Unis sur la nature de la technologie, des intellectuels japonais ont utilisé gijutsu pour réfléchir à apporter des solutions à certains des problèmes pressants de la modernité capitaliste. Des intellectuels de gauche, comme Aikawa Haruki (fl”川春喜), furent parmi les voix les plus notables dans un débat dont la tribune était la revue Études sur le matérialisme (Yuibutsuron kenkyû 唯物論研究). Bien que l'importance des techniques fût évidente, les ingénieurs étaient pratiquement absents des premiers débats, même si le mot « 
ingénieurs » (gishi 技 $\mathrm{fi}^{\mathrm{i}}$ ) est composé en japonais de deux caractères : 技 gi, pour les arts et les techniques, et fi' shi, pour le maître.

3 Le chapitre suivant détaille donc les efforts des ingénieurs pour participer aux discussions sur les techniques et pénètre ainsi les milieux des élites intellectuelles et politiques du Japon. Centrée sur Miyamoto Takenosuke, ingénieur renommé et principal architecte du mouvement social des ingénieurs, cette partie du livre suit la manière dont la profession a cherché à se positionner dans les débats en utilisant des concepts comme 《 point de vue de la technologie » (gijutsu no tachiba 技術の立場), 《 technologie globale » (sôgô gijutsu 総合技術) ou « technologie pour développer l'Asie ».

4 Le recours à ces concepts met en évidence leur lutte pour une meilleure position sociale et professionnelle dans l'administration publique dominée par les diplômés en droit. Pendant l'ère Meiji (1868-1912), les ingénieurs et les techniciens ont joué un rôle considérable dans la transformation sociale et matérielle du Japon, mais n'ont pas eu de visibilité sociale ni professionnelle, tant pour les succès que les échecs. Dans les années 1910 et 1920, leur présence dans les projets gouvernementaux a été plus importante et ils se sont joints aux débats, même si leur discours avait encore peu de résonnance. La reconnaissance de leur importance grandissante ne s'est pas traduite par une amélioration de leur statut dans la fonction publique, ni par celle de leur rémunération restée relativement basse. Cependant, durant les années 1920 et 1930, ingénieurs et bureaucrates-technologistes commencent à avancer une "définition explicitement technocratique de la technologie » comme principe d'organisation de la société japonaise et de son empire.

5 Le contraste entre les années 1920 et les années 1930 est révélateur des liens forts et constructifs entre l'empire japonais et ses ingénieurs. Dans les années 1920, inspiré par les efforts des ingénieurs américains pour promouvoir le statut de leur profession, Naoki Rintarô, chef du Bureau des rivières et ports de Tokyo, est l'un des premiers à propager l'idée d'« ingénieurs comme managers ». Attaquant l'idée de cantonner les ingénieurs à trouver les solutions techniques pour réaliser les ambitions des autres, Naoki propose que les techniques soient une pratique vivante, créative et humaine, consacrée à améliorer la vie des hommes : les ingénieurs doivent être essentiels non seulement pour concevoir et organiser, mais aussi pour exécuter et gérer. En 1920, inspiré par Naoki, Miyamoto Takenosuke crée le Club des ingénieurs (Kôjin kurabu 工人 クラブ). Soucieuse de promouvoir le statut des ingénieurs et de promouvoir la technologie comme force essentielle du développement social, l'organisation hésite entre organisation syndicale et association professionnelle. En 1928, les opposants à la variante syndicale prennent le dessus, mais leurs efforts pour ouvrir d'autres horizons aux ingénieurs et pour populariser la science et l'ingénierie s'intensifient, même s'ils n'ont qu'un succès limité.

Quand, en 1930, le gouvernement japonais commence à mobiliser l'industrie lourde pour soutenir ses aspirations coloniales en Chine, des ingénieurs acceptent d'aider à travailler au projet colonial et à lui donner une légitimité. S'ils n'avaient jusqu'alors pas véritablement réussi à s'imposer comme une élite en métropole, les ingénieurs réussissent à s'établir en Mandchourie et dans le nord de la Chine comme dirigeants et pionniers de la coopération sino-japonaise «basée sur le principe de la complémentarité » (p. 72). 
7 En d'autres termes, l'entreprise coloniale a donné un second souffle au concept d'ingénieurs-managers. Pour la plupart d'entre eux, leurs travaux en Chine ne se limitaient pas à une "invasion technologique " dans le but d'en exploiter les ressources. De leur point de vue, le Japon fournissait la technologie pour développer la prospérité commune et la paix éternelle en Asie orientale. Au contraire de la métropole japonaise où les ingénieurs étaient contraints par un système bureaucratique rigide qu'ils ne contrôlaient pas, en Chine, ils pouvaient plus librement utiliser leur expérience et mettre en œuvre un plan intégré de développement industriel. Le déclenchement de la guerre de 1938 a à peine changé le ton et les motifs de leur discours. L'idée de coopération a été remplacée par celle de fraternité et l'éloge des dirigeants chinois audacieux et visionnaires appelant à l'aide le Japon a été remplacé par la préoccupation pour les paysans chinois et leur besoin de la technologie japonaise pour les sauver des inondations, des famines et des déplacements forcés. Cet optimisme ne disparaitra qu'au début des années 1940 à la suite d'événements violents, tel que le sabotage des digues sur le Fleuve Jaune. Ces événements ont laissé Miyamoto et ses collègues ingénieurs désabusés.

8 La seconde partie de l'ouvrage examine les sites de cinq grands projets : l'aménagement de la rivière Liao en Mandchourie, le projet de planification urbaine pour Beijing, le projet de port et de zone industrielle d'Andong, et les barrages de Fengman et de Sup'ung. En observant ceux qu'il appelle " ingénieurs de projet » (plutôt que bureaucrates-technologistes), A. Moore illustre comment les acteurs sur le terrain ont développé et employé l'imaginaire technologique de l'empire japonais pendant la guerre. Autrement dit, la distinction est faite entre les ingénieurs des colonies en Chine et en Mandchourie et ceux qui travaillent à Taiwan ; la distinction est basée sur la transformation d'une économie coloniale centrée sur la commercialisation de la production agricole et minière dans ce que des historiens comme Kobayashi Hideo appellent « l'industrialisation militaire ». Ce changement a affecté non seulement le type de projets sur lesquels les ingénieurs japonais ont eu à travailler, mais aussi l'échelle à laquelle ils pouvaient développer leur imaginaire technologique.

9 Le soin donné à la phase d'implantation des projets a permis de pallier les limites de la conception et de laisser plus de liberté à leur imaginaire. Vers la fin des années 1930, nombre d'ingénieurs japonais sont partis en Chine pour la " sauver ». Une fois arrivés, ils ont été confrontés à un climat et à une géographie qu'ils ne connaissaient pas, mais qu'ils devaient maîtriser pour accomplir leur mission. Alors qu'ils s'enorgueillissaient d'apporter la technologie japonaise " moderne » à une Chine " arriérée ", leurs échecs les ont conduits à se tourner vers leurs collègues chinois. Bien qu'ils présentaient leurs projets de planification urbaine comme une modernisation d'une Chine en proie au chaos, le processus de construction entrepris s'est avéré désordonné et les changements de plans étaient la règle plutôt que l'exception. L'imaginaire faisait face à la réalité.

10 L'ouvrage de A. Moore est important dans le contexte de l'historiographie du Japon moderne car il est un des rares à mettre en exergue l'importance des techniques dans le projet de pan-asianisme, jusqu'alors examiné dans ses aspects politiques et culturels. Moore dévoile que l'expertise technique y était essentielle. L'ouvrage s'inscrit ainsi dans un courant historiographique récent qui considère que l'étude des techniques est un prisme clé pour éclairer le fonctionnement de l'empire japonais ${ }^{1}$. Pour les historiens 
des techniques et du colonialisme, Moore apporte un éclairage important sur la façon dont le terme " technologie " a été élaboré dans le cadre du projet impérialiste. Il montre comment une idée comme la " technologie globale " n'a pas été " pensée d'abord mais a émergé de Mandchourie en négociant des paysages et des climats mal connus, en acquérant de nouvelles connaissances et expertises en contact avec les techniciens chinois, en mettant en accord les objectifs (coloniaux) japonais en conflit d'industrialisation, de colonisation agricole et de contrôle des crues, et en faisant face à la résistance chinoise et à l'évolution des hostilités ". Finalement, la partie de l'ouvrage qui porte sur les barrages - principalement le chapitre 5 - est une contribution depuis longtemps attendue à une histoire globale des projets hydro-électriques, contribution que l'auteur semble vouloir approfondir encore ${ }^{2}$.

11 Le seul bémol à cet ouvrage est l'impression donnée par A. Moore que les ingénieurs ne sont apparus sur la scène historique qu'en 1920, avec la fondation du Club des ingénieurs. Mais, lorsque les voix de Miyamoto et Naoki commencent à s'exprimer au nom de leur profession, il y a déjà deux générations d'ingénieurs et de techniciens qui, avec des formations diverses et sous des appellations variées, ont exercé le métier. L'école d'ingénierie dont Miyamoto et Naoki étaient tous deux issus, l'École supérieure d'ingénierie (Kôbu daigakko 工部”学奢苂), avait été créée en 1873 et le premier Ordre des Ingénieurs (kôgakkai 工学会) avait tenu ses premières réunions en 1879. Dans les années 1890, il existait plusieurs organisations d'ingénieurs de diverses spécialités. La concentration sur les années 1920 et 1930 est justifiée par l'importance de l'entreprise coloniale dans l'organisation du livre. En même temps, l'émergence des ingénieurs en tant que groupe de praticiens dans le Japon moderne est une histoire qui reste encore à écrire.

De la même façon, étant donnée la continuité des acteurs dans le temps, l'étude aurait bénéficié d'en étendre les cadres chronologiques jusqu'aux années 1950, quand certains des acteurs essaient de se réinventer comme conseillers d'assistance technique dans le cadre des contrats de réparations et, au-delà, de s'insérer dans un réseau global de consultants qui se livrent une compétition pour obtenir des contrats non seulement en Asie orientale, mais aussi en Irak ou au Ghana.

\section{NOTES}

1. David G. Wittner, Technology and the culture of progress in Meiji Japan, New York, Routledge, 2008 ; Daqing Yang, Technology of Empire: telecommunications and Japanese expansion in Asia, 1883-1945, Cambridge, Harvard University Press, 2010.

2. Aaron Moore, "Japanese development consultancies and post-colonial power in Southeast Asia: the case of Balu Chaung hydropower project in Burma ", East Asia Science, Technology and Society, vol. 8, $\mathrm{n}^{\circ} 3$, September 2014 ; Aaron Moore, " The Yalu River era of developing Asia: Japanese expertise, colonial power and the construction of Sup'ung dam, 1937-1945 », Journal of Asian Studies, vol. 72, n 1, February 2013, p. 115-139. 


\section{AUTEURS}

ALEKSANDRA KOBILJSKI

UMR 8173 Chine, Corée, Japon, EHESS 